Mathematical Modelling and Analysis

Volume 17 Number 1, February 2012, 78-89 http://dx.doi.org/10.3846/13926292.2012.645076

(c) Vilnius Gediminas Technical University, 2012
Publisher: Taylor\&Francis and VGTU

http://www.tandfonline.com/TMMA

Print ISSN: 1392-6292

Online ISSN: 1648-3510

\title{
On Some Spectral Properties of Third Order Nonlinear Boundary Value Problems*
}

\section{Sergey Smirnov}

\section{Daugavpils University}

Parades str. 1, LV-5400 Daugavpils, Latvia

E-mail: srgsm@inbox.lv

Received August 29, 2011; revised November 22, 2011; published online February 1, 2012

\begin{abstract}
The present paper deals with a two point the third-order nonlinear boundary value problem. An estimation of the number of solutions to boundary value problem and their nodal structure are established. Some results are given on spectral properties of solutions.
\end{abstract}

Keywords: nonautonomous differential equations, nonlinear boundary value problems, multiplicity of solutions, nodal structure of solutions, spectral curves.

AMS Subject Classification: 34B15.

\section{Introduction}

In this paper we consider a boundary value problem of the form

$$
\begin{gathered}
x^{\prime \prime \prime}=-\lambda p(t) f(x), \\
x(0)=x^{\prime}(0)=0, \quad x(1)=0,
\end{gathered}
$$

where $\lambda$ is a positive parameter, functions $f(x)$ and $p(t)$ are continuous, $p(t) \geq 0, p(t) \not \equiv 0$. In what follows we shall assume that

(A1) $x f(x)>0$ for $x \neq 0$;

(A2) there exist $m, M>0$ such that $|f(x)|>M$ when $|x|>m$;

(A3) $\int_{t_{0}}^{+\infty} p(s) d s=\infty, \int_{-\infty}^{t_{0}} p(s) d s=\infty$.

These assumptions provide the oscillatory behavior of solutions of equation (1.1).

\footnotetext{
* This work has been supported by the European Social Fund within the project "Support for the implementation of doctoral studies at Daugavpils University", Agreement Nr. 2009/0140/1DP/1.1.2.1.2/09/IPIA/VIAA/015.
} 
We also use the following assumptions

(A4) $p(A t)=A^{k} p(t)$ for some $k \geq 0, A>0$;

(A5) $f(A x)=A^{q} f(x)$ for some $q>1, A>0$.

Conditions (A1) and (A2) are independent. It is easy to verify, that if conditions (A1) and (A5) are satisfied, then condition (A2) holds also. First, let chose $0<x_{1}<x_{2}$. Obviously, there exists $A>1$ such, that $x_{2}=A x_{1}$. Now consider $f\left(x_{2}\right)=f\left(A x_{1}\right)=A^{q} f\left(x_{1}\right)>f\left(x_{1}\right)$. Thus $f(x)$ is strictly increasing function for $x>0$.

Analogously we can show that $f(x)$ is strictly increasing for $x<0$. Let $x_{2}<x_{1}<0$. Then there exists $A>1$ such, that $x_{2}=A x_{1}$. Now consider $f\left(x_{2}\right)=f\left(A x_{1}\right)=A^{q} f\left(x_{1}\right)<f\left(x_{1}\right)$. Therefore, from conditions (A1) and (A5) (A2) follows. We state condition (A2) because some statements below hold if only (A1) and (A2) are satisfied for some function $f(x)$. Similarly we can show, that if condition (A4) is satisfied, then (A3) holds also.

An example of equation (1.1), which satisfies conditions (A1), (A2), (A3), (A4) and (A5) is $x^{\prime \prime \prime}=-\lambda t^{2} x^{3}$.

The special case $f(x)=|x|^{q} \operatorname{sgn} x, q>1$ is of a particular interest. In fact, the Emden-Fowler type differential equation

$$
x^{\prime \prime \prime}+p(t)|x|^{q} \operatorname{sgn} x=0
$$

is a prototype of (1.1). Therefore, equation (1.1) is a generalization of the second order Emden-Fowler equation

$$
u^{\prime \prime}+t^{\eta}|u|^{\mu} \operatorname{sgn} u=0,
$$

where $\eta, \mu$ are real constants. Such equations appear in the problems of polytropic gas spheres of finite radius or finite mass $[6,14]$. The study of EmdenFowler equation (1.4) has been one of the main objects in the field of nonlinear analysis in recent years since the appearance of the monograph due to R. Bellman [2].

The theory of nonlinear boundary value problems is an important and actual area of research since it is aimed to applications. Classical results in the theory concern the existence and uniqueness of solutions. A variety of techniques are employed in the theory, for example, methods that involve differential inequalities, shooting and angular function techniques, lower and upper solutions method, functional analytic approaches, topological methods, etc. Let us mention books by P. Bailey, L. Shampine, P. Waltman [10], S. Bernfeld and V. Lakshmikantham [3], N.I. Vasilyev and Yu.A. Klokov [16], C. de Coster and P. Habets [5], W. Kelley and A. Peterson [17].

Results concerning two point third order nonlinear boundary value problems were obtained by E. Rovderova [11], F. Sadyrbaev [12]. In [11] the author states some results on the number of solutions of two point boundary value problems. In [12] the author established multiplicity results for certain classes of third order nonlinear boundary value problems. His approach was based on the Hanan theory [9] of conjugate points for third order linear differential 
equations. Results which ensure the existence of infinitely many solutions of two point higher order nonlinear boundary value problem under the superlinear growth condition are given by C. de Coster and M. Gaudenzi [4].

On the other hand, there is voluminous literature concerning asymmetric nonlinearities. For example, investigation of the Fučik type equation of the second order ([13] and references therein), study of the third order asymmetric equations where the right side is a piece-wise linear function [7]. These types of investigations are motivated also by technical applications, for instance the theory of suspension bridges. Multiplicity of solutions for third order boundary value problem with asymmetric nonlinearity is established in [15]. A nonlinear spectra for the Neumann problem is investigated in [8].

The aim of the present paper is to provide results on the estimation of the number of solutions to boundary value problem (1.1), (1.2), their nodal structure and spectral properties. Therefore we are interested in solutions which have a double zero at $t=0$ and oscillate for $t>0$. The combination of the shooting method and scaling method is used for treating the number of solutions to boundary value problems.

The shooting method reduces solving a boundary value problem to solving of an initial value problem. So we consider the auxiliary initial value problem for the equation (1.1) with initial data

$$
x(0)=0, \quad x^{\prime}(0)=0, \quad x^{\prime \prime}(0)=\beta
$$

and we are looking for such $\beta$ that the solution of the corresponding initial value problem vanishes at $t=1$.

In view of the use of the shooting method, the problem the author faced with is the non-continuability of the solutions. For example, the function

$$
x(t)=(105 / 8)^{\frac{1}{2}}\left(t-t_{0}\right)^{-\frac{3}{2}}
$$

is a non-continuable solution of the equation $x^{\prime \prime \prime}=-x^{3}$ defined for $t>t_{0}$. Results concerning non-continuability of solutions of the third order nonlinear differential equations can be found in [1]. However, we will show that the noncontinuability does not influence the results on estimation of the number of solutions to boundary value problem.

The paper is organized as follows. Section 2 is devoted to the oscillatory properties of solutions of equation (1.1). In Section 3 we consider dependence of zeros of solutions on initial data. In Section 4 we deal with the number of solutions to boundary value problem (1.1), (1.2) and their spectral properties. Also one example is given to illustrate the results.

\section{Oscillatory Properties of Solutions}

In this section we discuss zero properties of solutions of equation (1.1). In the discussion we shall repeatedly use elementary arguments which, for the sake of convenience, we shall state as proposition. 
Proposition 1. Suppose $x(t) \in C^{3}(I)$. If $x(a) \geq 0, x^{\prime}(a) \leq 0, x^{\prime \prime}(a) \geq 0$ (but not all zero) and $x^{\prime \prime \prime}(t) x(t)<0$ when $x(t) \neq 0$, then $x(t)>0, x^{\prime}(t)<0$, $x^{\prime \prime}(t)>0$ for $t<a$.

Proof. Let $x(a) \geq 0, x^{\prime}(a) \leq 0, x^{\prime \prime}(a) \geq 0$ and $(x(a))^{2}+\left(x^{\prime}(a)\right)^{2}+\left(x^{\prime \prime}(a)\right)^{2}>0$. In all cases $x(t)$ will be positive in some open interval with the right boundary point $t=a$.

Suppose that there exists a point $t=t_{0}$ such that $x\left(t_{0}\right)=0$ and $x(t)>0$ for $t_{0}<t<a$. Since $x\left(t_{0}\right)=0$, there will exist a point $t=t_{1}, t_{0} \leq t_{1}<a$ such that $x^{\prime}\left(t_{1}\right)=0$ and there will exist a point $t=t_{2}, t_{0} \leq t_{2}<a$ such that $x^{\prime \prime}\left(t_{2}\right)=0$. Since $x^{\prime \prime \prime}(t) x(t) \leq 0$, it follows that $x^{\prime \prime \prime}(t)<0$ for $t_{0}<t<a$.

Consider

$$
x^{\prime \prime}(t)=x^{\prime \prime}(a)-\int_{t}^{a} x^{\prime \prime \prime}(s) d s, \quad t_{0} \leq t<a .
$$

The right-hand side is positive, and increases as $t \rightarrow-\infty$, as long as $x^{\prime \prime \prime}(t)$ remains negative. We thus conclude that $x^{\prime \prime}(t)$ is positive for $t_{0} \leq t<a$.

Consider

$$
x^{\prime}(t)=x^{\prime}(a)-\int_{t}^{a} x^{\prime \prime}(s) d s, t_{0} \leq t<a .
$$

The right-hand side is negative, and decreases as $t \rightarrow-\infty$, as long as $x^{\prime \prime}(t)$ remains positive. We thus conclude that $x^{\prime}(t)$ is negative for $t_{0} \leq t<a$.

Consider

$$
x(t)=x(a)-\int_{t}^{a} x^{\prime}(s) d s, \quad t_{0} \leq t<a .
$$

The right-hand side is positive, and increases as $t \rightarrow-\infty$, as long as $x^{\prime}(t)$ remains negative. We thus conclude that $x(t)$ is positive for $t_{0} \leq t<a$. These contradictions prove the proposition.

Corollary 1. Suppose $x(t) \in C^{3}(I)$. If $x(a) \leq 0, x^{\prime}(a) \geq 0, x^{\prime \prime}(a) \leq 0$ (but not all zero) and $x^{\prime \prime \prime}(t) x(t)<0$ when $x(t) \neq 0$, then $x(t)<0, x^{\prime}(t)>0, x^{\prime \prime}(t)<0$ for $t<a$.

Proof. The proof follows from Proposition 1 considering $y(t)=-x(t)$.

Remark 1 . The function $x(t)$ from Proposition 1 and Corollary 1 may be assumed to be a solution of differential equation (1.1).

An application of Proposition 1 and Corollary 1 leads to the following results.

Corollary 2. Assume that condition (A1) is satisfied. If $x(t)$ is a nontrivial solution of (1.1), $x(a)=x(b)=0$ and $a<b$, then $x^{\prime}(b) \neq 0$ (a simple zero cannot exist to the left of a double zero).

Proof. Let $x^{\prime}(b)=0$, and, without loss of generality, let $x^{\prime \prime}(b)>0$. Then, by Proposition $1 x(t)>0$ for $t<b$. But $x(a)=0, a<b$. The contradiction proves the corollary. 
Corollary 3. Suppose that condition (A1) holds. If $x(t)$ is a nontrivial solution of $(1.1)$ and $x(a)=x(b)=0(a<b)$, then $x^{\prime}(b) x^{\prime \prime}(b)>0$.

Proof. Assume $x^{\prime}(b) x^{\prime \prime}(b) \leq 0$. If $x^{\prime}(b)<0, x^{\prime \prime}(b) \geq 0$, then by Proposition 1 $x(t)>0$ for $t<b$. We have a contradiction, since $x(a)=0$. If $x^{\prime}(b)>0$, $x^{\prime \prime}(b) \leq 0$, then, by Corollary $1 x(t)<0$ for $t<b$. We have a contradiction, since $x(a)=0$. In view of Corollary $2, x^{\prime}(b) \neq 0$.

Proposition 2. Let $x(t)$ be a solution of equation (1.1) such that $x(a)=$ $x^{\prime}(b)=0(a<b), x(t) \neq 0$ for $t \in(a, b)$. If condition (A1) is fulfilled, then $x(t)$ vanishes in $(b,+\infty)$.

Proof. Assume that $x(t)$ does not change sign for $t>b$. Without loss of generality, let $x(t)>0, t>b$. Multiplying the equation (1.1) by $x(t)$ and integrating from $a$ to $t$, we obtain

$$
\int_{a}^{t} x(s) x^{\prime \prime \prime}(s) d s=-\lambda \int_{a}^{t} x(s) p(s) f(x(s)) d s .
$$

Integrating the first term by parts, we get

$$
x(t) x^{\prime \prime}(t)-x(a) x^{\prime \prime}(a)-\int_{a}^{t} x^{\prime \prime}(s) x^{\prime}(s) d s=-\lambda \int_{a}^{t} x(s) p(s) f(x(s)) d s,
$$

or

$$
x(t) x^{\prime \prime}(t)=\frac{1}{2} x^{\prime 2}(t)-\frac{1}{2} x^{\prime 2}(a)-\lambda \int_{a}^{t} x(s) p(s) f(x(s)) d s .
$$

If $t=b$ we obtain

$$
x(b) x^{\prime \prime}(b)=\frac{1}{2} x^{\prime 2}(b)-\frac{1}{2} x^{\prime 2}(a)-\lambda \int_{a}^{b} x(s) p(s) f(x(s)) d s<0 .
$$

Since $x(b)>0$, then $x^{\prime \prime}(b)<0$. Since $x(t)>0$, then (in view of (A1) and (1.1)) $x^{\prime \prime \prime}(t)<0$ and $x^{\prime \prime}(t)$ is strictly decreasing. Thus, $x^{\prime \prime}(t)<0$ for $t>b$ and $x^{\prime}(t)$ is strictly decreasing for $t>b$. Since $x^{\prime}(b)=0$ and $x^{\prime}(t)$ is strictly decreasing for $t>b$, then $x^{\prime}(t)<0$ for $t>b$. Thus, $x(t)$ is strictly decreasing for $t>b$. If two consecutive derivatives of $x(t)$ are negative then $x(t)$ must ultimately be negative. This completes the proof of the proposition.

Proposition 3. Let $x(t)$ be a solution of equation (1.1) such that $x(a)=0$. If conditions (A1), (A2) and (A3) hold, then $x(t)$ vanishes in $(a,+\infty)$.

Proof. Suppose that $x(t)$ does not vanish for $t>a$. Without loss of generality, let $x(t)>0$ for $t>a$. If there exists $b>a$ such that $x^{\prime}(b)=0$, then the proof follows from the Proposition 2 above. Therefore, assume that $x^{\prime}(t)$ does not vanish for $t>a$. Since $x^{\prime}(t)>0$ for $t$ immediately to the right of $a$, it follows that $x^{\prime}(t)>0$ for $t>a$. As $x(t)>0$, then (in view of (A1) and (1.1)), $x^{\prime \prime \prime}(t)<0$ and $x^{\prime \prime}(t)$ is strictly decreasing. 
First suppose there exists $t_{1} \geq a$ such that $x^{\prime \prime}\left(t_{1}\right)=0$. Then $x^{\prime \prime}(t)<0$ for $t>t_{1}$. If two consecutive derivatives of $x^{\prime}(t)$ are negative then $x^{\prime}(t)$ must ultimately be negative.

Now assume that $x^{\prime \prime}(t)>0$ for $t>a$. So $x^{\prime}(t)$ is strictly increasing for $t>a$. Integrating equation (1.1) between $t_{0}>a$ and $t$ we obtain

$$
\int_{t_{0}}^{t} x^{\prime \prime \prime}(s) d s=-\lambda \int_{t_{0}}^{t} p(s) f(x(s)) d s
$$

or eliminating nonnegative terms and using assumption (A2) we get

$$
x^{\prime \prime}\left(t_{0}\right)=x^{\prime \prime}(t)+\lambda \int_{t_{0}}^{t} p(s) f(x(s)) d s \geq \lambda \int_{t_{0}}^{t} p(s) f(x(s)) d s \geq M \lambda \int_{t_{0}}^{t} p(s) d s .
$$

The left side is independent of $t$ and thus the integral on the right hand side must converge as $t \rightarrow+\infty$. This contradiction proves the proposition.

Remark 2. The condition $x(a)=0$ in Proposition 3 is essential, because there exist solutions (for example (1.5)) of equation (1.1) which do not vanish.

Corollary 4. Assume that conditions (A1), (A2) and (A3) fulfil. If $x(t)$ is a nontrivial solution of (1.1) and $t=a$ is a zero of $x(t)$, then $x(t)$ has an infinity of simple zeros in $(a,+\infty)$. If $t=a$ is a double zero of $x(t)$, then $x(t)$ does not vanish in $(-\infty, a)$.

Remark 3. Suppose that $x(t)$ is a nontrivial solution of equation (1.1) with double zero at point $t=0\left(x(0)=x^{\prime}(0)=0\right)$. There exists an increasing sequence of points $0<t_{1}^{\prime \prime}<t_{1}^{\prime}<t_{1}<t_{2}^{\prime \prime}<t_{2}^{\prime}<t_{2}<\cdots$ such that $x\left(t_{i}\right)=$ $x^{\prime}\left(t_{i}^{\prime}\right)=x^{\prime \prime}\left(t_{i}^{\prime \prime}\right)=0(i=1,2, \ldots)$.

Proposition 4. It is true, that only one of the functions $x(t), x^{\prime}(t)$ or $x^{\prime \prime}(t)$ vanishes at each of the points $t_{i}, t_{i}^{\prime}, t_{i}^{\prime \prime}(i=1,2, \ldots)$.

Proof. Let us consider $x\left(t_{i}\right)=x^{\prime \prime}\left(t_{i}\right)=0$ and without loss of generality, let $x^{\prime}\left(t_{i}\right)>0$. Then, by Proposition $1 x(t)>0$ for $t<t_{i}$. But $x(0)=0,0<t_{i}$. We get the contradiction. The other cases can be treated in analogous way.

The points $t_{i}, t_{i}^{\prime}, t_{i}^{\prime \prime}(i=1,2, \ldots)$ are called nodal points.

Next consider sequences of values $x(t), x^{\prime}(t)$ and $x^{\prime \prime}(t)$ at the nodal points. There are six such sequences $\left(\left|x^{\prime}\left(t_{i}\right)\right|\right),\left(\left|x^{\prime \prime}\left(t_{i}\right)\right|\right),\left(\left|x\left(t_{i}^{\prime}\right)\right|\right),\left(\left|x^{\prime \prime}\left(t_{i}^{\prime}\right)\right|\right),\left(\left|x\left(t_{i}^{\prime \prime}\right)\right|\right)$, $\left(\left|x^{\prime}\left(t_{i}^{\prime \prime}\right)\right|\right)$ and propositions below investigate their behavior. In the statements below we prove that the above sequences of absolute values are increasing. It is plausible also that the sequences $\left(x^{\prime}\left(t_{i}\right)\right),\left(x^{\prime \prime}\left(t_{i}\right)\right),\left(x\left(t_{i}^{\prime}\right)\right),\left(x^{\prime \prime}\left(t_{i}^{\prime}\right)\right),\left(x\left(t_{i}^{\prime \prime}\right)\right)$, $\left(x^{\prime}\left(t_{i}^{\prime \prime}\right)\right)$ are alternating. The idea of the proof of these six propositions is taken from [1].

The first two propositions deal with the values of first and second derivatives at the zeros of solution.

Proposition 5. If conditions (A1), (A2) and (A3) are fulfilled, then the sequence $\left(\left|x^{\prime}\left(t_{i}\right)\right|\right)$ is strictly increasing. 
Proof. Let $t_{1}$ and $t_{2}$ be arbitrary consecutive zeros of $x(t)$. As $x f(x)>0$ (in view of $(\mathrm{A} 1))$, then $x x^{\prime \prime \prime}<0$. Therefore

$$
\begin{aligned}
0 & >\int_{t_{1}}^{t_{2}} x \cdot x^{\prime \prime \prime} d t=\mid \text { integrating by parts }\left|=x x^{\prime \prime}\right|_{t_{1}}^{t_{2}}-\int_{t_{1}}^{t_{2}} x^{\prime} x^{\prime \prime} d t \\
& =-\int_{t_{1}}^{t_{2}} x^{\prime} d x^{\prime}=-\frac{x^{\prime}\left(t_{2}\right)^{2}}{2}+\frac{x^{\prime}\left(t_{1}\right)^{2}}{2} .
\end{aligned}
$$

Thus, $x^{\prime}\left(t_{1}\right)^{2}<x^{\prime}\left(t_{2}\right)^{2}$ or $\left|x^{\prime}\left(t_{1}\right)\right|<\left|x^{\prime}\left(t_{2}\right)\right|$.

Proposition 6. If condition (A1) is fulfilled, $f(x)$ is strictly increasing and differentiable, $p(t) \equiv p>0$, then the sequence $\left(\left|x^{\prime \prime}\left(t_{i}\right)\right|\right)$ is strictly increasing.

Proof. Let $t_{1}$ and $t_{2}$ be arbitrary consecutive zeros of $x(t)$. Consider

$$
\begin{aligned}
\int_{t_{1}}^{t_{2}} x^{\prime \prime} x^{\prime \prime \prime} d t & =-\int_{t_{1}}^{t_{2}} x^{\prime \prime} \lambda p f(x) d t=-\lambda p \int_{t_{1}}^{t_{2}} x^{\prime \prime} f(x) d t \\
& =-\left.\lambda p x^{\prime} f(x)\right|_{t_{1}} ^{t_{2}}+\lambda p \int_{t_{1}}^{t_{2}} x^{\prime 2} f^{\prime}(x) d t=\lambda p \int_{t_{1}}^{t_{2}} x^{\prime 2} f^{\prime}(x) d t>0 .
\end{aligned}
$$

On the other hand

$$
\int_{t_{1}}^{t_{2}} x^{\prime \prime} x^{\prime \prime \prime} d t=\int_{t_{1}}^{t_{2}} x^{\prime \prime} d x^{\prime \prime}=\frac{x^{\prime \prime}\left(t_{2}\right)^{2}}{2}-\frac{x^{\prime \prime}\left(t_{1}\right)^{2}}{2} .
$$

Thus, $x^{\prime \prime}\left(t_{1}\right)^{2}<x^{\prime \prime}\left(t_{2}\right)^{2}$ or $\left|x^{\prime \prime}\left(t_{1}\right)\right|<\left|x^{\prime \prime}\left(t_{2}\right)\right|$.

The next two propositions deal with the values of solution and its second derivative at the zeros of the first derivative.

Proposition 7. If $f(x)=|x|^{q} \operatorname{sgn} x, q>1$ and $p(t) \equiv p>0$, then the sequence $\left(\left|x\left(t_{i}^{\prime}\right)\right|\right)$ is strictly increasing.

Proof. Let $t_{1}^{\prime}$ and $t_{2}^{\prime}$ be arbitrary consecutive zeros of $x^{\prime}(t)$. Consider

$$
\int_{t_{1}^{\prime}}^{t_{2}^{\prime}} x^{\prime} x^{\prime \prime \prime} d t=\left.x^{\prime} x^{\prime \prime}\right|_{t_{1}^{\prime}} ^{t^{\prime}}-\int_{t_{1}^{\prime}}^{t_{2}^{\prime}} x^{\prime \prime 2} d t=-\int_{t_{1}^{\prime}}^{t_{2}^{\prime}} x^{\prime \prime 2} d t<0 .
$$

On the other hand

$$
\begin{aligned}
\int_{t_{1}^{\prime}}^{t_{2}^{\prime}} x^{\prime} x^{\prime \prime \prime} d t & =-\lambda p \int_{t_{1}^{\prime}}^{t_{2}^{\prime}} x^{\prime}|x|^{q} \operatorname{sgn} x d t=-\lambda p \int_{t_{1}^{\prime}}^{t_{2}^{\prime}}|x|^{q} \operatorname{sgn} x d x \\
& =-\frac{\lambda p}{q+1}\left(\left|x\left(t_{2}^{\prime}\right)\right|^{q+1}-\left|x\left(t_{1}^{\prime}\right)\right|^{q+1}\right)<0 .
\end{aligned}
$$

Thus $\left|x\left(t_{2}^{\prime}\right)\right|^{q+1}>\left|x\left(t_{1}^{\prime}\right)\right|^{q+1}$ or $\left|x\left(t_{2}^{\prime}\right)\right|>\left|x\left(t_{1}^{\prime}\right)\right|$.

Proposition 8. If condition (A1) is fulfilled, $f(x)$ is strictly increasing and differentiable, $p(t) \equiv p>0$, then the sequence $\left(\left|x^{\prime \prime}\left(t_{i}^{\prime}\right)\right|\right)$ is strictly increasing. 
Proof. Let $t_{1}^{\prime}$ and $t_{2}^{\prime}$ be arbitrary consecutive zeros of $x^{\prime}(t)$. Consider

$$
\begin{aligned}
\int_{t_{1}^{\prime}}^{t_{2}^{\prime}} x^{\prime \prime} x^{\prime \prime \prime} d t & =-\int_{t_{1}^{\prime}}^{t_{2}^{\prime}} x^{\prime \prime} \lambda p f(x) d t=-\lambda p \int_{t_{1}^{\prime}}^{t_{2}^{\prime}} x^{\prime \prime} f(x) d t \\
& =-\left.\lambda p x^{\prime} f(x)\right|_{t_{1}^{\prime}} ^{t^{\prime}}+\lambda p \int_{t_{1}^{\prime}}^{t_{2}^{\prime}} x^{\prime 2} f^{\prime}(x) d t=\lambda p \int_{t_{1}^{\prime}}^{t_{2}^{\prime}} x^{\prime 2} f^{\prime}(x) d t>0 .
\end{aligned}
$$

On the other hand

$$
\int_{t_{1}^{\prime}}^{t_{2}^{\prime}} x^{\prime \prime} x^{\prime \prime \prime} d t=\int_{t_{1}^{\prime}}^{t_{2}^{\prime}} x^{\prime \prime} d x^{\prime \prime}=\frac{x^{\prime \prime}\left(t_{2}^{\prime}\right)^{2}}{2}-\frac{x^{\prime \prime}\left(t_{1}^{\prime}\right)^{2}}{2} .
$$

Thus, $x^{\prime \prime}\left(t_{1}^{\prime}\right)^{2}<x^{\prime \prime}\left(t_{2}^{\prime}\right)^{2}$ or $\left|x^{\prime \prime}\left(t_{1}^{\prime}\right)\right|<\left|x^{\prime \prime}\left(t_{2}^{\prime}\right)\right|$.

The next two propositions investigate the values of solution and its first derivative at the zeros of the second derivative.

Proposition 9. If $f(x)=|x|^{q} \operatorname{sgn} x, q>1$ and $p(t) \equiv p>0$, then the sequence $\left(\left|x\left(t_{i}^{\prime \prime}\right)\right|\right)$ is strictly increasing.

Proof. Let $t_{1}^{\prime \prime}$ and $t_{2}^{\prime \prime}$ be arbitrary consecutive zeros of $x^{\prime \prime}(t)$. Consider

$$
\int_{t_{1}^{\prime \prime}}^{t_{2}^{\prime \prime}} x^{\prime} x^{\prime \prime \prime} d t=\left.x^{\prime} x^{\prime \prime}\right|_{t_{1}^{\prime \prime}} ^{t_{2}^{\prime \prime}}-\int_{t_{1}^{\prime \prime}}^{t_{2}^{\prime \prime}} x^{\prime \prime 2} d t=-\int_{t_{1}^{\prime \prime}}^{t_{2}^{\prime \prime}} x^{\prime \prime 2} d t<0
$$

On the other hand

$$
\begin{aligned}
\int_{t_{1}^{\prime \prime}}^{t_{2}^{\prime \prime}} x^{\prime} x^{\prime \prime \prime} d t & =-\lambda p \int_{t_{1}^{\prime \prime}}^{t_{2}^{\prime \prime}} x^{\prime}|x|^{q} \operatorname{sgn} x d t=-\lambda p \int_{t_{1}^{\prime \prime}}^{t_{2}^{\prime \prime}}|x|^{q} \operatorname{sgn} x d x \\
& =-\frac{\lambda p}{q+1}\left(\left|x\left(t_{2}^{\prime \prime}\right)\right|^{q+1}-\left|x\left(t_{1}^{\prime \prime}\right)\right|^{q+1}\right)<0 .
\end{aligned}
$$

Thus $\left|x\left(t_{2}^{\prime \prime}\right)\right|^{q+1}>\left|x\left(t_{1}^{\prime \prime}\right)\right|^{q+1}$ or $\left|x\left(t_{2}^{\prime \prime}\right)\right|>\left|x\left(t_{1}^{\prime \prime}\right)\right|$.

Proposition 10. If conditions (A1), (A2) and (A3) are fulfilled, then the sequence $\left(\left|x^{\prime}\left(t_{i}^{\prime \prime}\right)\right|\right)$ is strictly increasing.

Proof. Let $t_{1}^{\prime \prime}$ and $t_{2}^{\prime \prime}$ be arbitrary consecutive zeros of $x^{\prime \prime}(t)$. Consider

$$
\int_{t_{1}^{\prime \prime}}^{t_{2}^{\prime \prime}} x^{\prime} x^{\prime \prime} d t=\left.x x^{\prime \prime}\right|_{t_{1}^{\prime \prime}} ^{t^{\prime \prime}}-\int_{t_{1}^{\prime \prime}}^{t_{2}^{\prime \prime}} x x^{\prime \prime \prime} d t>0
$$

On the other hand

$$
\int_{t_{1}^{\prime \prime}}^{t_{2}^{\prime \prime}} x^{\prime} x^{\prime \prime} d t=\int_{t_{1}^{\prime \prime}}^{t_{2}^{\prime \prime}} x^{\prime} d x^{\prime}=\frac{x^{\prime}\left(t_{2}^{\prime \prime}\right)^{2}}{2}-\frac{x^{\prime}\left(t_{1}^{\prime \prime}\right)^{2}}{2}>0
$$

or $x^{\prime}\left(t_{2}^{\prime \prime}\right)^{2}>x^{\prime}\left(t_{1}^{\prime \prime}\right)^{2}$. Thus $\left|x^{\prime}\left(t_{2}^{\prime \prime}\right)\right|>\left|x^{\prime}\left(t_{1}^{\prime \prime}\right)\right|$. 


\section{Dependence of Zeros on Initial Data}

Proposition 11. Suppose that conditions (A4) and (A5) are fulfilled. If $x(t)$ is a solution of equation (1.1) with $\lambda \equiv 1$, such that

$$
x(0)=x^{\prime}(0)=0, \quad x^{\prime \prime}(0)=\gamma \neq 0,
$$

then every solution of equation (1.1) with arbitrary $\lambda$ which has a double zero at $t=0$ and the second derivative at $t=0$ of the same sign as $\gamma$ can be expressed via solution $x(t)$ as

$$
y(t)=B^{\frac{3+k}{q-1}} x(B t) / \lambda^{\frac{1}{q-1}},
$$

with initial data

$$
y(0)=y^{\prime}(0)=0, \quad y^{\prime \prime}(0)=B^{\frac{3+k}{q-1}+2} \gamma / \lambda^{\frac{1}{q-1}},
$$

where $B>0$ is a parameter.

Remark 4. We distinguish the cases of $x^{\prime \prime}$ having opposite signs at $t=0$ in Proposition 11, because if $x(t)$ is a solution of $(1.1)$, then $(-x(t))$ need not $\mathrm{o}$ be a solution of (1.1). As an example we present the equation

$$
x^{\prime \prime \prime}=-\left\{\begin{array}{ll}
2 x^{3}, & x \geq 0, \\
x^{3}, & x<0
\end{array},\right.
$$

where $f(x)$ satisfies condition (A5) with $q=3$.

Remark 5. A similar statement for higher order Emden-Fowler type autonomous equation can be found in [1].

Proof. The proposition can be proved by direct substitution. So

$$
y^{\prime \prime \prime}(t)=B^{\frac{3+k}{q-1}+3} x^{\prime \prime \prime}(B t) / \lambda^{\frac{1}{1-q}}, \quad f(y(t))=B^{\frac{3+k}{q-1} q} f(x(B t)) / \lambda^{\frac{1}{q-1} q} .
$$

Then

$$
\begin{aligned}
\frac{B^{\frac{3+k}{q-1}+3}}{\lambda^{\frac{1}{1-q}}} x^{\prime \prime \prime}(B t) & =-\lambda p(t) \frac{B^{\frac{3+k}{q-1} q}}{\lambda^{\frac{1}{q-1} q}} f(x(B t)) \\
& =-\lambda p(B t) B^{-k} \frac{B^{\frac{3+k}{q-1} q}}{\lambda^{\frac{q}{q-1}}} f(x(B t))=\lambda B^{-k} \frac{B^{\frac{3+k}{q-1} q}}{\lambda^{\frac{q}{q-1}}} x^{\prime \prime \prime}(B t) .
\end{aligned}
$$

Therefore

$$
\frac{B^{\frac{3+k}{q-1}+3}}{\lambda^{\frac{1}{1-q}}}=\lambda B^{-k} \frac{B^{\frac{3+k}{q-1} q}}{\lambda^{\frac{q}{q-1}}}, \quad \text { or } \quad \frac{B^{\frac{3 q+k}{q-1}}}{\lambda^{\frac{1}{1-q}}}=\frac{B^{\frac{3 q+k}{q-1}}}{\lambda^{\frac{1}{1-q}}} .
$$

Hence the proof.

Now we assume that conditions (A1), (A2) and (A3) are satisfied. Let $y(t)$ be a nontrivial solution of equation (1.1) with the initial conditions $y(0)=0$, $y^{\prime}(0)=0, y^{\prime \prime}(0)=\beta$. Let us denote simple zeros of $y(t)$ to the right from $t_{0}=0$ by $t_{1}, t_{2}, \ldots, t_{i}, \ldots$. The next statement follows from Proposition 11. 
Corollary 5. Suppose that conditions (A4) and (A5) are fulfilled and let $\lambda$ in equation (1.1) be fixed. If $\beta$ continuously and monotonically tends to $\pm \infty$ (resp. to zero), then $t_{i}$ continuously and monotonically tend to zero (resp. to $+\infty)$.

Proof. If $\beta$ continuously and monotonically tends to $\pm \infty$ (zero), then, by Proposition 11, $B$ continuously and monotonically tends to $+\infty$ (zero). Thus, $t_{i}$ continuously and monotonically tend to $t=0( \pm \infty)$.

\section{Number of Solutions and Spectral Curves}

Theorem 1. Suppose that conditions (A1), (A4) and (A5) are fulfilled, $\lambda$ is fixed, then boundary value problem (1.1), (1.2) has a countable set of solutions $x_{i}(t)$ with $x_{i}^{\prime \prime}(0)>0$ and a countable set of solutions $u_{i}(t)$ with $u_{i}^{\prime \prime}(0)<0$, $i=0,1,2, \ldots$. Any solution $x_{i}(t)$ and $u_{i}(t)$ has exactly $i$ simple zeros in $(0,1)$.

Proof. Consider auxiliary initial value problem (1.1), $x(0)=x^{\prime}(0)=0$, $x^{\prime \prime}(0)=\beta$. By Proposition 11 and Corollary 5 we can choose $|\beta|$ so small, that $t_{1}>1$, and $|\beta|$ so large, that for any fixed $i$ one has $t_{i}<1$.

Remark 6 . Theorem 1 remains valid if boundary conditions (1.2) are replaced by more general ones

$$
x(0)=0, \quad x^{(i)}(0)=0, \quad x^{(j)}(1)=0, \quad i \in\{1,2\}, j \in\{0,1,2\} .
$$

Remark 7. It follows from Theorem 1 above, that solutions to boundary value problem (1.1), (1.2) has a specific nodal structure. There exist exactly two solutions of $(1.1),(1.2)$ which do not vanish in $(0,1)$, the first one is positive in $(0,1)$ and the second one is negative in $(0,1)$. There exist exactly two solutions of $(1.1),(1.2)$ which vanish in $(0,1)$ exactly once, the first one has positive second derivative at $t=0$ and the second one has negative second derivative at $t=0$. And generally, for every $i=0,1,2, \ldots$ there exist exactly two solutions of $(1.1),(1.2)$ which vanish in $(0,1)$ exactly $i$ times, the first one has positive second derivative at $t=0$ and the second one has negative second derivative at $t=0$.

Remark 8. It follows from Theorem 1 above, that boundary value problem (1.1), (1.2) with $\lambda \equiv 1$ has a countable set of solutions $x_{i}(t)$ with $x_{i}^{\prime \prime}(0)>0$ and a countable set of solutions $u_{i}(t)$ with $u_{i}^{\prime \prime}(0)<0, i=0,1,2, \ldots$ Any solution $x_{i}(t)$ and $u_{i}(t)$ has exactly $i$ simple zeros in $(0,1)$. Let denote $x_{i}^{\prime \prime}(0)=\beta_{i}^{+}>0$ and $u_{i}^{\prime \prime}(0)=\beta_{i}^{-}<0, i=0,1,2, \ldots$ Therefore, there exist an increasing sequence of values $\beta_{i}^{+}>0$ and decreasing sequence of values $\beta_{i}^{-}<0$, such that boundary value problem $(1.1),(1.2)$ has nontrivial solutions with exactly $i$ simple zeros in $(0,1)$.

Now we consider the problem of finding a set of parameters $(\lambda, \beta)$, where $\beta=x^{\prime \prime}(0)$ such that problem (1.1), (1.2) has nontrivial solutions. We will show that a set of $(\lambda, \beta)$ with the described property consists of spectral curves. The 
spectral curves for the problem consist of infinite set of branches $L_{i}^{+}$and $L_{i}^{-}$ $(i=0,1,2, \ldots)$ which can be obtained analytically and graphically. The lower index shows how many zeros has the respective solution in the interval $(0,1)$, and the upper index shows the sign of the second derivative of a solution at $t=0$.

Theorem 2. The spectral curves for problem (1.1), (1.2) are the branches given by

$$
L_{i}^{+}=\left\{(\lambda, \beta): \beta=\frac{\beta_{i}^{+}}{\lambda^{\frac{1}{q-1}}}\right\}, \quad L_{i}^{-}=\left\{(\lambda, \beta): \beta=\frac{\beta_{i}^{-}}{\lambda^{\frac{1}{q-1}}}\right\}, \quad i=0,1, \ldots
$$

Proof. Let $x_{i}(t)$ be a solution of boundary value problem (1.1), (1.2) with $\lambda \equiv 1$, which has exactly $i$ simple zeros in $(0,1), i=0,1, \ldots$ Consider

$$
y_{i}(t)=B^{\frac{3+k}{q-1}} x_{i}(B t) / \lambda^{\frac{1}{q-1}} .
$$

In view of Proposition $11 y_{i}(t)$ is solution of equation (1.1), with double zero at $t=0$. If $B=1$, then $y_{i}(t)$ has zeros at the same points as $x_{i}(t)$ has, and hence $y_{i}(t)$ is solution of boundary value problem (1.1), (1.2). Thus, it follows from Proposition 11, that

$$
\beta=\beta_{i}^{+} \lambda^{-\frac{1}{q-1}} \quad \text { or } \quad \beta=\beta_{i}^{-} \lambda^{-\frac{1}{q-1}} \text {. }
$$

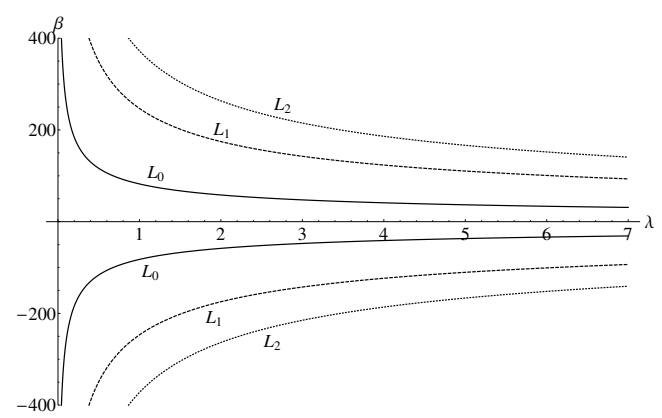

Figure 1. Some spectral curves for problem (4.1). $L_{0}$ - solid, $L_{1}$ - dashed, $L_{2}-$ dotted.

Example 1. Consider the problem

$$
x^{\prime \prime \prime}=-\lambda t^{2} x^{3}, \quad x(0)=x^{\prime}(0)=0, \quad x(1)=0 .
$$

First suppose that $\lambda \equiv 1$ and find $\beta_{i}^{+}$and $\beta_{i}^{-}$. Since the nonlinearity $f(x)=x^{3}$ is symmetric, then $\beta_{i}^{+}=-\beta_{i}^{-}=\beta_{i}$. Thus, by using numerical simulation, we obtain

$$
\beta_{0}=82.26, \quad \beta_{1}=246.85, \quad \beta_{2}=372.33 \text {. }
$$

So, by Theorem 2, we get

$$
L_{0}: \beta= \pm 82.26 / \sqrt{\lambda}, \quad L_{1}: \beta= \pm 246.85 / \sqrt{\lambda}, \quad L_{2}: \beta= \pm 372.33 / \sqrt{\lambda} .
$$

See Figure 1, where some spectral curves for problem (4.1) are presented. 


\section{References}

[1] I.V. Astashova. Application of dynamical systems to the study of asymptotic properties of solutions to nonlinear higher-order differential equations. J. Math. Sci. Springer Science+Business Media, 126:1361-1391, 2005.

[2] R. Bellman. Stability Theory of Differential Equations. McGraw-Hill, New York, 1953.

[3] S. Bernfeld and V. Lakshmikantham. An Introduction to Nonlinear Boundary Value Problems. Academic Press, New York, 1974.

[4] C. de Coster and M. Gaudenzi. On the existence of infinitely many solutions for superlinear $n$-th order boundary value problems. Nonlinear World, 4:505-524, 1997.

[5] C. de Coster and P. Habets. Two-Point Boundary Value Problems: Lower and Upper Solutions. Elsevier Science, 2006.

[6] R. Emden. Gaskugeln. Leipzig, 1907.

[7] M. Gaudenzi and P. Habets. Fučik spectrum for a third order equation. J. Differential Equations, 128:556-595, 1996. http://dx.doi.org/10.1006/jdeq.1996.0106.

[8] A. Gritsans and F. Sadyrbaev. Nonlinear spectra: the Neumann problem. Math. Model. Anal., 14(1):33-42, 2009. http://dx.doi.org/10.3846/1392-6292.2009.14.33-42.

[9] M. Hanan. Oscillation criteria for third order linear differential equation. Pacific J. Math., 11:919-944, 1961.

[10] P. Waltman P. Bailey, L. Shampine. Nonlinear Two Point Boundary Value Problems. Academic Press, New York, 1968.

[11] E. Rovderova. On the number of solutions of a third-order boundary-value problem. Trans. Amer. Math. Soc., 347:3079-3092, 1995. http://dx.doi.org/10.2307/2154772.

[12] F. Sadyrbaev. Multiplicity results for third order two-point boundary value problems. Proceedings of the University of Latvia. Mathematics. Differential equations, 616:5-16, 1999.

[13] F. Sadyrbaev and A. Gritsans. Nonlinear spectra for parameter dependent ordinary differential equations. Nonlinear Anal. Model. Control, 12(2):253-267, 2007.

[14] G. Sansone. Equazioni differenziali nel campo reale. Parte seconda. Bologna, 1949.

[15] S. Smirnov. On the third order boundary value problems with asymmetric nonlinearity. Nonlinear Anal. Model. Control, 16(2):231-241, 2011.

[16] N.I. Vasilyev and Yu.A. Klokov. Foundations of the Theory of Boundary Value Problems for Ordinary Differential Equations. Zinatne, Riga, 1978.

[17] A. Peterson W. Kelley. The Theory of Differential Equations. Classical and Qualitative. Pearson Education, 2004. 